Nurs Outlook. 2012 May ; 60(3): 143-148.e1. doi:10.1016/j.outlook.2011.10.002.

\title{
Altruism in Clinical Research: Coordinators' Orientation to their Professional Roles
}

\author{
Jill A. Fisher, Ph.D. ${ }^{1}$ [Assistant Professor] and Corey A. Kalbaugh, M.S., M.A. ${ }^{2}[$ Doctoral \\ Student] \\ ${ }^{1}$ Center for Biomedical Ethics \& Society, Vanderbilt University, Nashville, TN 37203 \\ 2Department of Epidemiology, Gillings School of Global Public Health, University of North \\ Carolina at Chapel Hill, Chapel Hill, NC 27599-7435
}

\section{Abstract}

\begin{abstract}
Background-Research coordinators have significant responsibilities in clinical trials that often require them to find unique ways to manage their jobs, thus re-shaping their professional identities.
\end{abstract}

\begin{abstract}
Purpose-The purpose of this study is to identify how research coordinators manage role and ethical conflicts within clinical research trials.
\end{abstract}
Method-A qualitative study combining observation and 63 semi-structured interviews at 25 research organizations was used.

Discussion-Altruism is a recurring theme in how research coordinators define and view their work.

Conclusion-Altruism is adopted by research coordinators: 1) to teach patient-subjects the appropriate reasons to participate in clinical research, 2) to minimize the conflict between research and care, and 3) to contest the undervaluation of coordinating. Altruism is a strategy employed to handle the various conflicts they experience in a difficult job, and it has become part of the professional identity of clinical research coordinators.

\section{Introduction}

Discussions about the role of altruism in clinical trials tend to focus on the participation of patients as research subjects. Altruism has been stressed as an important and appropriate motivator for individuals to enroll in research studies, with some even arguing that clinical trials are more ethical when participants act out of altruism instead of self-interest. ${ }^{1,2}$ In spite of the attention to participants' orientation to clinical research, little scholarly attention has focused on the role of altruism in shaping the roles or identities of researchers. ${ }^{3-5}$ In this paper, we argue that research coordinators - also known as study or trial coordinators and study nurses - are clinical research professionals for whom altruism is an essential part of their professional identity.

\footnotetext{
(C) 2011 Mosby, Inc. All rights reserved.

Corresponding Author: Jill A. Fisher, Ph.D., Center for Biomedical Ethics \& Society, Vanderbilt University, Nashville, TN 37203 , 2525 West End Ave., Suite 400, Phone: 615-936-2609, Fax: 615-936-3800, Jill.Fisher@ Vanderbilt.Edu.

Publisher's Disclaimer: This is a PDF file of an unedited manuscript that has been accepted for publication. As a service to our customers we are providing this early version of the manuscript. The manuscript will undergo copyediting, typesetting, and review of the resulting proof before it is published in its final citable form. Please note that during the production process errors may be discovered which could affect the content, and all legal disclaimers that apply to the journal pertain.
} 
Research coordinators are critical to the success of clinical trials, yet much of their labor is invisible compared to principal investigators (PIs) who tend to receive the bulk of attention as researchers. ${ }^{6}$ Although coordinators have less authority in clinical research than do PIs, they often have greater daily responsibilities. ${ }^{7-9}$ Coordinators manage many aspects of clinical trials: setting up and conducting study protocols, recruiting and retaining patients in studies, and acting as point persons for sponsors and institutional review boards (IRBs). ${ }^{10}$

A key finding from our empirical research on the organization of clinical trials work is that coordinators mobilize altruism as a means of managing their interactions with participants, PIs, and others. This paper will focus on the various ways that altruism helps coordinators to cope with 1) the task of motivating participants to adhere to study protocols, 2) the tension between research and care, and 3) the undervaluation of their work in the research enterprise. Our findings suggest that an altruistic orientation to research can become integrated into coordinators' identities with, we argue, problematic implications for the profession.

\section{Background}

Research is a complex clinical activity. Studies are funded - or sponsored - by multiple sources, including the institutions where they are conducted, large government agencies such as the National Institutes of Health (NIH), non-profit foundations such as the American Cancer Society, and industry such as pharmaceutical companies. Depending on the type of research being conducted, there are many titles and types of research staff who are involved in the clinic. In spite of differences, there tend to be two critical roles in research: the principal investigator and research coordinator. PIs - who are usually physicians but also are nurses and other professionals - design the studies or are contracted to provide study oversight. PIs are also generally responsible for taking medical histories, conducting physical exams, and making final determinations about the eligibility of patients for clinical trials. Depending on the type of study and the individual investigator, PIs often delegate the majority of the research activities to research coordinators. ${ }^{11}$ In some cases, PIs are so absent from the day-to-day activities of clinical research that they have been referred to as "phantom investigators."

Research coordinators, in turn, have the responsibility of managing the clinical trials to which they are assigned. This usually means overseeing participant recruitment; timeline enforcement - such as study visits, procedures, and self-report instruments - for each participant; data entry via source documents and case report forms; and relationship maintenance with the study sponsor, the investigators they work for, the IRBs reviewing the ethics of the studies, and the participants in the trials. It should be noted that specialized research positions sometimes support research coordinators' work, including personnel for recruitment, regulatory affairs, business development, and quality assurance. In small research organizations, however, coordinators are responsible for the work of all of these positions.

There are a substantial number of activities for coordinators to manage in clinical research. For instance, Papke conducted a survey of coordinators and identified 128 different activities that are part of their routine functions. ${ }^{14}$ Furthermore, Davis et al. identified a series of nineteen different general skill types with twenty-five subcategories that are needed for effective research coordination. ${ }^{6}$ Others have noted that, of all research staff members, coordinators are most likely to perceive ethical dilemmas in the treatment of participants or observe research misconduct on the part of PIs. ${ }^{4,5,15}$ The multifaceted responsibilities given to coordinators can be overwhelming and can quickly lead to stress, especially as the number of research studies to which they have been assigned increases. ${ }^{4,8,16}$ 
As well, coordinators have been shown to experience a variety of role conflicts in their work. Based on a qualitative study of coordinators' role in the ethical conduct of research, Davis et al. described three advocacy roles (i.e., patient, subject, study) that have contradictory commitments and create potential conflicts. ${ }^{6}$ For example, coordinators must juggle their responsibility to the best interest of individual participants with their responsibility to the larger interests of the science in which they are participating. Other scholars have written about this conflict as the "science/care dilemma" in clinical research. ${ }^{5,17}$ As the dominant group of coordinators, nurses are especially prone to experience this dilemma. ${ }^{18,19}$

In spite of these concerns, one must not dismiss the important role that nurses as nurses play in research. ${ }^{9}, 20,21$ Xanthos et al. have noted, "A holistic, caring approach fostered by nurses not only results in successful management of a complex protocol but also humanizes the research process for participants." 22 Likewise, Davis et al. found that coordinators perceived both caring and detachment as necessary in the research process. ${ }^{6}$ Hence, it is important to determine how coordinators, particularly nurse coordinators, deal with the potential conflicts in their jobs.

\section{Methods}

This paper draws upon qualitative research on how clinical trials are organized and executed in diverse clinical settings in the U.S. Through an institutional ethnography, the study investigated the everyday work lives of those engaging in clinical trials, paying particular attention to the role and ethical conflicts that were described by informants (e.g., PIs, coordinators, and research participants) and observed in their practices (e.g., recruitment of participants, informed consent processes, and study compliance). This research consisted of interviews and observation at 25 medical research organizations in two large cities in the southwestern U.S. The clinics at which observation and interviews were conducted included private practices, dedicated research sites, large hospitals, and not-for-profit clinics. The majority of the clinics conducted clinical trials for the pharmaceutical industry, and some received funding from multiple sources including industry, the $\mathrm{NIH}$, and private foundations.

The research clinics were identified using an online database, and all sites in two urban regions of the Southwest were contacted by telephone to participate in interviews or to consent to observation. No incentives were offered to promote participation, but the study was explained in detail by phone and again in person as part of the informed consent process. At least one individual agreed to an interview or observation in $75 \%$ of the clinics in one city and $50 \%$ of the clinics in the other. Based on information available online, there was no discernable difference between the clinics that agreed and refused to participate based on types of studies conducted, size of the research operation, or experience of the site.

Participant observation in clinics was focused primarily on interactions between the PIs and participants, as well as between coordinators and participants. Observation at sites ranged from 1-day visits to multiple visits spanning several months. In semi-structured interviews, interviewees were asked questions about their job responsibilities and their "typical day" as well as questions about their experiences working in clinical research, how research had changed over time, and what types of changes they would like to see in the future. All the data collection was done by one of the authors (initials removed for peer review), who is a white woman trained in sociological methods and was in her late $20 \mathrm{~s}$ at the time of the research. 
Interviews were completed with 63 individuals who were working in clinical research (i.e., PIs, coordinators, research administrators, recruiters, other research staff, and study monitors) or who were volunteering as participants in clinical studies. When possible, interviewees were clustered to get the perspective of multiple employees and participants at each clinic. Interviews were conducted until saturation was reached. Most relevant to this paper, we focus here on the experiences of the research coordinators interviewed $(n=18)$. The sample of coordinators consisted of 15 women and 3 men, of whom 16 were white and 2 Hispanic. Their ages ranged from late-twenties to late-sixties, with the majority being in their forties. Their level of experience as coordinators ranged widely from as little as three months to over 15 years. Interviews lasted approximately forty-five minutes. All interviews were recorded, transcribed, and stripped of personal identifiers. Informants were given the opportunity to edit their interview transcript before data were analyzed. The identities of all clinics and individuals included in the study are confidential. The study was reviewed and approved by the Institutional Review Board of Rensselaer Polytechnic Institute.

In keeping with a methodology of grounded theory, the data analysis relied on a multistaged process of coding field notes and interviews for core and emerging categories. Coding was multi-staged in order to revisit the data multiple times for depth of analysis and for the creation of cross-references among the data and the categories coded. The process of coding was done by creating detailed memos at the conclusion of clinic visits and by individually adding to the coding through the process of repeated, fine-grained reading of transcripts and observational notes for additional themes that emerged as important. During coding at the conclusion of data collection, we added more subtle codes that aimed to create sub-categories within emergent themes. We also were attentive to and coded for issues of organizational dynamics and power, like those influenced by role conflicts and gender. One important theme that we found - altruism - emerged through this analysis and is the focus of this paper.

\section{Results: Coordinators' Different Uses of "Altruism"}

"We [coordinators] are women who are dedicated to wanting the world to be better for our children [and] our grandchildren." (Interview, Research Coordinator)

In our research, altruism - either the explicit use of the term or more implicit occurrences of the concept - was a common theme in how research coordinators described the purpose and meaning of their work. This section describes different manifestations of altruism that we found in how coordinators talk about their positions or perform their jobs. We found that altruism has three functions: 1) to motivate participants to be adherent research subjects by underscoring the "right" reasons to participate in clinical trials, especially their contribution to science and society, 2) to minimize tensions they experience in this work between research and care, and 3) to contest the undervaluation of their work. Previous scholarship has illustrated that altruism is often gendered feminine, ${ }^{23,24}$ and we found a clear gender connotation with the concept in the concrete manifestations of altruism in the everyday lives of the coordinators we interviewed and observed.

\section{Motivating Participants to be Adherent Research Subjects}

Most research participants in clinical trials are not motivated to enroll for altruistic reasons. Instead, they tend to have more instrumental reasons, such as access to health care for those without adequate insurance or hope for a magic bullet for those with diseases without effective treatments. ${ }^{4}$ There is a strong sense among many of the coordinators in our study that patients volunteering to participate in drug studies should be altruistic as well, even if altruism is not their initial motivation to enroll. Teaching altruism, then, becomes part of the coordinators' goal when enrolling patients into studies. One coordinator explained: 
There are very few people that enter our studies that are altruistic, except at the end then they really become altruistic because we try to teach them what research is about, it's not about being a hamster and you joined a study. You want to learn... why this is done, what the principle behind it is, and at the end, they're like "Wow, I really helped some other people." "Yeah, you have." But they didn't [think] that, typically, going in.

Coordinators envision altruism to be an effective way of not only helping patients enroll in clinical trials for the "right" reasons but also of encouraging their adherence with the study protocols. This is because coordinators often note that participants must have a deep appreciation for the goals of research in order to make good, adherent research subjects.

Unlike standard medical care in which patients' adherence is largely an individualized problem, researchers and sponsors are relying on subjects to follow the study protocols strictly as a way of producing reliable data and information about new therapies. Many coordinators believe that clinical trial participants need to volunteer selflessly. Otherwise, they point out, elements of the studies could be viewed as too cumbersome or difficult. For example, a coordinator said,

Because a clinical trial is giving of yourself: your time, collecting data, you're asking them to write in diaries, to do things that are perhaps many times outside of their normal schedule, to come in earlier, to stay longer for office visits... We want a patient who's inquisitive and who wants to know more about the study, about the medication that they're going to be consuming, about the trials process, about what their responsibilities are, and you want a person who's going to give you the kind of data that will either show that particular compound should never go on the market or that this compound will definitely make an impact in people's lives.

Of course, coordinators hope there will also be some personal benefit to the individual patient-subjects participating in the study. Nonetheless, from their perspective, altruistic participants are going to make better study subjects because they understand that they have to give of themselves for the good of medicine and science. Because participants ordinarily do not have this perspective at enrollment, some coordinators view this as teaching altruism to participants.

\section{Minimizing the Conflict Between Research and Care}

Coordinators can experience profound role conflicts because of the competing goals associated with research and care. ${ }^{5,17}$ In our sample, coordinators seemed most distressed about what has been characterized as the "therapeutic misconceptions" that research participants often form. ${ }^{25,}{ }^{26}$ Specifically, there is a sense among coordinators that participants do not, or cannot, differentiate what it means to be part of a research study from what they have learned to expect from standard medical care. A coordinator shared one of her experiences:

An example would be the participant we had that was doing this [study] for psoriasis. It was unfortunate that out of the four people that have [enrolled in the study], he was the one [whose condition] was the worst and had been getting worse - which was why he came in. Well, we were almost sure he got the placebo. He got no effect... Even though he'd read the informed consent [form] and we'd explained it to him, he didn't understand it well: "How would they pick me to not get the drug when I'm so bad?'... And even if you think you've made it clear, it isn't always clear.

The difference between research and care is not only a distinction that is difficult for participants to grasp, but coordinators confess to struggling with prioritizing the goals of 
research over the best therapeutic outcomes for individual participants. It is difficult for them to see participants' conditions fail to improve or even get worse due to the investigational drug or from receiving a placebo during the course of a clinical trial. Yet, keeping these participants in the studies is important for the results of the clinical trial. For example, a coordinator commented, "Unfortunately, we have some studies right now that are not a good option. For me, it's difficult when I have a conflict between whether this is really the best thing for the subject or not."

As a strategy for coping with the role conflict they experience in the research context, coordinators frequently understand their work in altruistic terms: that they are helping to advance scientific knowledge and to develop treatments in medicine that will eventually benefit patients and society. Even when individual participants are not helped by clinical trials, coordinators often talk about the broader impacts that their work has had on the practice of medicine. As one coordinator explained,

When we first started, we did studies with Bextra ${ }^{\circledR}$ before it was approved by the FDA. We did studies on Levitra ${ }^{\circledR}$ before that was approved. And right now, I've been told that we have two or three studies that we worked on, where the pharmaceutical company has just now submitted their application to the FDA for a new drug, which is really exciting for us.

Many coordinators are active in tracking the progress of new therapies even after the studies are over in order to keep track of the "successes" of their clinic because they feel pride in and even some ownership of the products they had a part in bringing to market. By reframing their work in this way, an altruistic narrative is used to justify why individual participants might not get the best, individualized care they need through clinical trials. This re-framing reduces some of the conflict between research and care coordinators experience.

\section{Contesting the Undervaluation of Coordinating}

Because research is structured by the traditional dynamics of the doctor-nurse relationship, the PI-coordinator relationship has the same rigid hierarchy of value and credit. Namely, the work that coordinators do is seen as requiring less expertise than what is required of PIs. Beyond that distinction, however, is the perception - which is often associated with nursing as well - that the skills that coordinators bring to their positions are "natural" attributes of all women, such as strength in interpersonal communication, empathy, and multi-tasking. ${ }^{27-29}$ This effect is exacerbated by the fact that most PIs are men (82\%) and most coordinators $(90 \%)$ are women. ${ }^{7}$ The gendering of a profession as "women's work" tends to lead to undervaluation in the forms of diminished pay and constraints on professional advancement. ${ }^{27}$ This is the pattern in clinical research in spite of efforts to create certification programs for research coordinators to distinguish those with training and experience in their roles. ${ }^{4}$

Compared to current trends in nursing, coordinators seem to be more likely to embrace gender as a primary part of their professional identities. A final manifestation of altruism in coordinators' narratives is re-framing their work as a "higher calling" that is invaluable to the clinical research enterprise whether or not it is recognized as such. Specifically, a recurring theme in interviews was their maternal approach to their work. As one coordinator asserted, "We do a lot of handholding. Sometimes we get accused of being mothers. But a lot of [participants], they do become our brothers or our sons." Overall, many coordinators feel that an altruistic orientation to their work is a critical part of "care" in clinical trials. As one coordinator asserted, "that's that personal element - that as much as it is science and we use the word 'subjects' and 'protocols' and things - they're people and you know I can't get around that." 
The linking of altruism to gendered notions of care - typified in references to mothering indicates that coordinators' identities are linked to being part of a predominantly female profession that is undervalued. Like the ethic of care that is often associated with nursing and motherhood, ${ }^{30-33}$ the adoption of altruism in coordinating adds a higher calling to an often tedious and taken-for-granted job. As historically has been the case with nursing, altruism adds a sense of purpose because it emphasizes, for coordinators, the feminized, maternal characteristics that cannot be replaced, or at least not replaced by (male) PIs. ${ }^{34}$

\section{Limitations}

As with all research, our study has limitations. Although we included a diverse sample of research organizations in our study, the number of coordinators interviewed was fairly small. In addition, our study was not designed to investigate altruism per se, so we did not systematically document how an altruistic orientation might differ based on therapeutic area, study design, or a host of other variables. To better understand how altruism functions within clinical research, future studies could probe not only how altruism manifests in coordinators work but areas in which this orientation is absent or de-emphasized.

Nonetheless, given that the coordinators in our sample described the importance of altruism in an unprompted way, we believe that the results of our study represent an under-theorized phenomenon in clinical research. Importantly, our study illustrates that altruism should not be thought of as merely an attitude toward research but rather as a crucial, yet problematic, adaptation to a stressful and difficult job.

\section{Conclusion}

Altruism has long been a characteristic associated with the nursing profession. ${ }^{35,} 36$ Yet in the context of research, an element of "selfish altruism" as described by Haigh is apparent. ${ }^{37}$ In other words, coordinators' altruistic orientation is not so much in the best interest of the patient-subject as it is an acceptable mechanism to maintain a nursing identity in a professional environment that challenges a more traditional care paradigm (in Haigh's terminology it helps "ensure survival of the group"). Moreover, altruism is being leveraged in study participants in such a way that simultaneously improves coordinators' job performance by creating moral pressure for participants to be adherent to the study protocol. This double-move of embracing and teaching altruism enables coordinators to accept that some participants will receive a placebo as part of a clinical trial and that a lack of improvement in their conditions is not at odds with being a nurse. While this may be positive for individual coordinators, the mobilization of altruism is also problematic. On one hand, it minimizes the potential for coordinators to act as advocates for research participants, hiding the extent to which coordinators serve their organization or research sponsors instead of their patients. ${ }^{38}$ On the other, positioning altruism as a key feature of coordinators' identity further genders the profession, which tends to discount the expertise, skills, and knowledge they bring to their positions. ${ }^{7}$

In summary, research coordinators are vital to a robust and efficient system of clinical research. They are the protocol managers, participant educators, and liaisons for the sponsors and IRB. Through an examination of narratives of altruism, we see one aspect of how coordinators manage their various roles. Coordinators teach altruism to participants as a means of encouraging adherence to complex study protocols and retention for the length of trials. They also use altruism to cope with the research versus care dilemma in which participants are entrusted to their "care" but not in the traditional sense of the patient-nurse relationship. Finally, coordinators implement a gendered notion of altruism that is tied to motherhood and other feminine ideals as a way of counteracting the undervaluing of their work and skills. Hence, through adopting altruism in these various ways, clinical research 
coordinators continue to shape and re-shape their professional identities as a means of meeting the demands of a challenging profession.

\section{Acknowledgments}

Funding Information: The research was supported by the U.S. National Institutes of Health under Ruth L. Kirschstein National Research Service Award 5F31MH070222 from the National Institute of Mental Health. Its contents are solely the responsibility of the authors and do not necessarily represent the official views of the National Institutes of Health.

\section{References}

1. Jansen LA. The Ethics of Altruism in Clinical Research. Hastings Center Report. 2009; 39(4):2636. [PubMed: 19711632]

2. Williams B, Entwistle V, Haddow G, Wells M. Promoting research participation: why not advertise altruism? Social Science \& Medicine. Apr; 2008 66(7):1451-6. [PubMed: 18222579]

3. King NMP. Benefits, Harms, and Motives in Clinical Research. Hastings Center Report. 2009; 39(4):3. [PubMed: 19711622]

4. Fisher, JA. Medical Research for Hire: The Political Economy of Pharmaceutical Clinical Trials. Rutgers University Press; New Brunswick: 2009.

5. Fisher JA. Coordinating 'ethical' clinical trials: the role of research coordinators in the contract research industry. Sociology of Health \& Illness. 2006; 28(6):678-94. [PubMed: 17184412]

6. Davis AM, Hull SC, Grady C, WIlfond BS, Henderson GE. The Invisible Hand in Clinical Research: The study coordinator's critical role in human subjects protection. Journal of Law, Medicine \& Ethics. 2002; 30(3):411-9.

7. Fisher JA. Re-inscribing Gender in New Modes of Medical Expertise: The Investigator-Coordinator Relationship in the Clinical Trials Industry. Gender, Work \& Organization. 2010; 17(2):150-73.

8. Cantor AB, Roberts C, Johnson DJ, Gwede CK. Burnout in Clinical Research Coordinators in the United States. Oncology Nursing Forum. 2005; 32(6):1123-30. [PubMed: 16270108]

9. Grady C, Edgerly M. Science, Technology, and Innovation: Nursing Responsibilities in Clinical Research. Nursing Clinics of North America. 2009; 44(4):471-81. [PubMed: 19850183]

10. Woodin, KE. CRC's Guide to Coordinating Clinical Research. CenterWatch; Boston: 2004.

11. Ginsberg, D. Becoming a Successful Clinical Research Investigator. Thomson CenterWatch; Boston: 2005.

12. Gamache V. Chasing Away Phantom Investigators. CenterWatch Monthly. 2001; 8(11):1-5.

13. Redman BK, Templin TN, Merz JF. Research Misconduct Among Clinical Trial Staff. Science and Engineering Ethics. 2006; 12:481-9. [PubMed: 16909150]

14. Papke A. The ACRP National Job Analysis of the Clinical Research Coordinator. The Monitor. 1996:45-53.

15. Habermann B, Broome M, Pryor ER, Ziner KW. Research Coordinators' Experiences With Scientific Misconduct and Research Integrity. Nursing Research. 2010; 59(1):51-7. [PubMed: 20010045]

16. Spilsbury K, Petherick E, Cullum N, Nelson A, Nixon J, Mason S. The role and potential contribution of clinical research nurses to clinical trials. J Clin Nurs. 2007; 17:549-57. [PubMed: 17419791]

17. Mueller, M-R. Science versus care: physicians, nurses and the dilemma of clinical research. In: Elston, MA., editor. The Sociology of Medical Science and Technology. Blackwell; Oxford: 1997.

18. Mueller M-R. The Nurse Clinical Trial Coordinator: Benefits and Drawbacks of the Role. Research and Theory for Nursing Practice: An International Journal. 2002; 16(1):33-42.

19. Oberle K, Allen M. Ethical considerations for nurses in clinical trials. Nurs Ethics. 2006; 13(2): 180-6. [PubMed: 16526151]

20. Poston RD, Buescher CR. The essential role of the clinical research nurse (CRN). Urologic Nursing. Jan-Feb;2010;30(1):55-63. 77. 
21. Mori C, Mullen N, Hill EE. Describing the role of the clinical research nurse. Research Practitioner. 2007; 8(6):220-8.

22. Xanthos GJ, Carp D, Geromanos K. Recognizing nurses' contributions to the clinical research process. Journal of the Association of Nurses in AIDS Care. Jan-Feb;1998 9(1):39-48. [PubMed: 9436166]

23. Badgett MVL, Folbre N. Assigning Care: Gender Norms and Economic Outcomes. International Labour Review. 1999; 138(3):311-26.

24. Groome A. The Social Construction of Nursing. Contemporary Nurse. 1992; 1(1):44-7. [PubMed: 1596617]

25. Appelbaum PS, Lidz CW, Grisso T. Therapeutic Misconception in Clinical Research: Frequency and Risk Factors. IRB: Ethics \& Human Research. 2004; 26(2):1-8. [PubMed: 15069970]

26. Lidz CW, Appelbaum PS, Grisso T, Renaud M. Therapeutic Misconception and the Appreciation of Risks in Clinical Trials. Social Science \& Medicine. 2004; 58:1689-97. [PubMed: 14990370]

27. Statham, A.; Miller, EM.; Mauksch, HO. The Worth of Women's Work: A Qualitative Synthesis. State University of New York Press; Albany: 1988.

28. Radsma J. Caring and Nursing: a Dilemma. Journal of Advanced Nursing. 1994; 20(3):444-9. [PubMed: 7963048]

29. Speedy S. Feminism and the Professionalisation of Nursing. The Australian Journal of Advanced Nursing. 1987; 4(2):20-8. [PubMed: 3647783]

30. Kuhse, H. Caring: Nurses, Women and Ethics. Blackwell; Cambridge, MA: 1997.

31. Duffy M. Reproducing Labor Inequalities: Challenges for Feminists Conceptualizing Care at the Intersections of Gender, Race, and Class. Gender \& Society. 2005; 19(1):66-82.

32. Crittenden, A. The Price of Motherhood: Why the Most Important Job in the World is Still the Least Valued. Holt; New York: 2001.

33. Smith A. An analysis of altruism: A concept of caring. Journal of Advanced Nursing. 1995; 22:785-90. [PubMed: 8708200]

34. Reverby, S. Ordered to Care: the Dilemma of American Nursing, 1850-1945. Cambridge University Press; New York: 1987.

35. Fagermoen MS. Professional identity: values embedded in meaningful nursing practice. Journal of Advanced Nursing. 1997; 25:434-41. [PubMed: 9080267]

36. Kramer M. Comparative study of characteristics, attitudes and opinions of neophyte British and American nurses. International Journal of Nursing Studies. 1967; 4:281-94.

37. Haigh CA. Reconstructing nursing altruism using a biological evolutionary framework. Journal of Advanced Nursing. Jun; 2010 66(6):1401-8. [PubMed: 20384639]

38. Padgett SM. Dilemmas of Caring in a Corporate Context: A Critique of Nursing Case Management. Advances in Nursing Science. 1998; 20(4):1-12. [PubMed: 9595170] 